\title{
Current status of nontuberculous mycobacterial surgery in Japan: analysis of data from the annual survey by the Japanese Association for Thoracic Surgery
}

\author{
Yuji Shiraishi $^{1}$
}

Received: 5 August 2015/Accepted: 24 September 2015/Published online: 3 October 2015

(C) The Author(s) 2015. This article is published with open access at Springerlink.com

\begin{abstract}
Objective The prevalence of pulmonary disease caused by nontuberculous mycobacteria (NTM) has been increasing in Japan. Adjuvant resectional surgery is often recommended to lessen disease progression when the response to drug therapy is poor. In all likelihood, as affected cases of NTM disease increase, so will the number of operations. The goal of this study was to determine the current status of NTM surgery in Japan by analyzing data from the annual survey of the Japanese Association for Thoracic Surgery (JATS).

Methods Data were obtained from annual surveys conducted between 2008 and 2012. The annual number of operations for pulmonary NTM disease was tabulated nationwide and in each region (Hokkaido, Tohoku, Kanto, Tokyo, Chubu, Kinki, Chugoku/Shikoku, and Kyushu). For comparison, the numbers for pulmonary tuberculosis and tuberculoma operations were also obtained.

Results The annual number of operations for pulmonary NTM disease nationwide increased each year between 2008 and 2012: 292 (2008), 323 (2009), 452 (2010), 440 (2011), and 514 (2012); an overall increase of $76 \%$. Conversely, the annual numbers of operations for pulmonary tuberculosis were stable: 145 (2008), 181 (2009), 117 (2010), 113 (2011), and 107 (2012), as were the annual numbers of operations for tuberculoma: 386 (2008), 341 (2009), 320 (2010), 390 (2011), and 351 (2012).
\end{abstract}

Presented at the 68th Annual Scientific Meeting of The Japanese Association for Thoracic Surgery.

Yuji Shiraishi

yujishi@mvb.biglobe.ne.jp

1 Section of Chest Surgery, Fukujuji Hospital, 3-1-24 Matsuyama, Kiyose, Tokyo 204-8522, Japan
Conclusion Data from the JATS annual survey demonstrate a steady increase in the number of NTM surgeries in Japan. General thoracic surgeons will continue to increasingly encounter NTM patients who are candidates for surgery until a magic bullet against NTM disease is available.

Keywords Nontuberculous mycobacteriosis .

Tuberculosis $\cdot$ Tuberculoma $\cdot$ Resectional surgery

\section{Introduction}

Since the advent of potent anti-tuberculous drugs, the prevalence of pulmonary tuberculosis has been decreasing in Japan. In contrast, it is thought that the prevalence of pulmonary disease caused by nontuberculous mycobacteria (NTM) has been increasing. The estimated incidence rate of pulmonary NTM disease in Japan has increased from 1.5 per 100,000 in 1985 to 3.52 in 1997 [1]. NTM disease is usually indolent, but can result in extensive parenchymal destruction, causing respiratory failure and vulnerability to airway infection, which can be fatal [2]. According to the statement published by the American Thoracic Society (ATS)/Infectious Diseases Society of America (IDSA) in 2007, the primary treatment for pulmonary NTM disease is chemotherapy using a multidrug regimen [3]. However, chemotherapy has limited efficacy in pulmonary diseases caused by the majority of NTM species. Therefore, the statement proposed a multidisciplinary treatment approach, i.e., combined multidrug treatment regimen and adjuvant resectional surgery, for patients with intractable pulmonary NTM disease. The Japanese Society for Tuberculosis (JST) published guidelines for surgical intervention of pulmonary NTM disease in 2008 , which recommended a 
multidisciplinary treatment approach consistent with the 2007 ATS/IDSA statement [4]. The higher the prevalence of pulmonary NTM disease, the more likely the number of operations for this disease will increase. This study was designed to clarify the current status of NTM surgery in Japan by analyzing data from the annual survey of the Japanese Association for Thoracic Surgery (JATS).

\section{Materials and methods}

Data from the JATS annual survey conducted between 2008 and 2012 were used in this analysis. The year 2008 was used as a starting point because it was the year that survey questionnaires added the category of pulmonary NTM disease. The number of survey questionnaire forms that were sent out to officially certified institutions nationwide and their response rates in each year were as follows: $785 / 95.3 \%$ in 2008 [5], 798/96.5 \% in 2009 [6], $787 / 97.2 \%$ in 2010 [7], 790/95.6\% in 2011 [8], and $802 / 96.9 \%$ in 2012 [9]. The total numbers of general thoracic operations in each year were 61,315 in 2008 [5], 65,897 in 2009 [6], 67,960 in 2010 [7], 69,223 in 2011 [8], and 72,899 in 2012 [9]. The annual number of operations over the 5-year period for pulmonary NTM disease, regionally (Hokkaido, Tohoku, Kanto, Tokyo, Chubu, Kinki, Chugoku/Shikoku, and Kyushu) and nationwide, was extracted from the JATS annual survey database that comprises anonymized raw data. Data on the total number of operations for pulmonary tuberculosis and tuberculoma were also obtained for comparison.

\section{Results}

Nationally, the number of operations for pulmonary NTM disease in 2012 increased $76 \%$ over the number performed in 2008 (Table 1). The ratio of the number of operations for pulmonary NTM disease to the total number of general thoracic operations also increased from $0.5 \%(292 / 61,315)$ in 2008 to $0.7 \%(514 / 72,899)$ in 2012 . Regionally, an increase in NTM surgeries was particularly noted in Tohoku, Kanto, Tokyo, Chubu, Kinki, and Chugoku/Shikoku. In contrast, the annual number of operations performed for pulmonary tuberculosis (Table 2) and tuberculoma (Table 3) was stable during the 5-year period.

\section{Discussion}

Nontuberculous mycobacteria are environmental organisms found in soil and water that can produce opportunistic lung infections. The true incidence and prevalence of pulmonary NTM disease remains unclear because this disease is not communicable and has not been designated a reportable disease by the Ministry of Health, Labour, and Welfare in Japan. However, reports suggest that the prevalence of pulmonary NTM disease has been increasing $[1,2]$. Of $120+$ species of mycobacteria classified as NTM, only a few are known to lead to pulmonary disease [3]. In Japan, members of the Mycobacterium avium complex (MAC) are the most common source of pulmonary NTM disease [10]. Mycobacterium kansasii is the second most common NTM, but the incidence of $M$. kansasii pulmonary disease is much lower than that of pulmonary MAC disease [10]. Furthermore, a M. kansasii pulmonary infection is the only NTM disease that can be successfully treated with a multidrug regimen alone [3, 10]. Therefore, in daily clinical practice, physicians are more likely to be consulted about surgical intervention for pulmonary MAC disease.

The problem is that there is no specific agent for the treatment of pulmonary MAC disease. Although the ATS has recommended a multidrug regimen employing the newer macrolides (clarithromycin or azithromycin), rifampicin or rifabutin, ethambutol and, if necessary, injectable aminoglycoside (streptomycin) [11], the efficacy of this program remains limited. Kobashi and Matsushima reported that the combined therapy for pulmonary MAC disease recommended in the ATS [11] and the JST [12] guidelines produced unsatisfactory results compared to the
Table 1 Annual trend of number of national and regional operations for pulmonary nontuberculous mycobacterial disease

\begin{tabular}{lccccc}
\hline Region & $2008[5]$ & $2009[6]$ & $2010[7]$ & $2011[8]$ & $2012[9]$ \\
\hline Hokkaido & 12 & 18 & 26 & 16 & 13 \\
Tohoku & 16 & 19 & 37 & 28 & 39 \\
Kanto & 54 & 62 & 96 & 72 & 86 \\
Tokyo & 33 & 57 & 53 & 72 & 90 \\
Chubu & 40 & 58 & 69 & 83 & 78 \\
Kinki & 72 & 45 & 68 & 67 & 95 \\
Chugoku/Shikoku & 32 & 46 & 60 & 52 & 75 \\
Kyushu & 33 & 18 & 43 & 50 & 38 \\
Nation (total) & 292 & 323 & 452 & 440 & 514 \\
\hline
\end{tabular}


Table 2 Annual trend of number of national and regional operations for pulmonary tuberculosis

\begin{tabular}{lccccc}
\hline Region & $2008[5]$ & $2009[6]$ & $2010[7]$ & $2011[8]$ & $2012[9]$ \\
\hline Hokkaido & 5 & 13 & 6 & 8 & 2 \\
Tohoku & 4 & 4 & 4 & 6 & 1 \\
Kanto & 20 & 33 & 29 & 20 & 23 \\
Tokyo & 20 & 42 & 13 & 23 & 19 \\
Chubu & 8 & 19 & 17 & 9 & 14 \\
Kinki & 49 & 44 & 19 & 27 & 23 \\
Chugoku/Shikoku & 18 & 12 & 11 & 10 & 8 \\
Kyushu & 21 & 14 & 18 & 10 & 17 \\
Nation (total) & 145 & 181 & 117 & 113 & 107 \\
\hline
\end{tabular}

Table 3 Annual trend of number of national and regional operations for tuberculoma

\begin{tabular}{lccccc}
\hline Region & $2008[5]$ & $2009[6]$ & $2010[7]$ & $2011[8]$ & $2012[9]$ \\
\hline Hokkaido & 24 & 34 & 17 & 18 & 14 \\
Tohoku & 30 & 30 & 38 & 28 & 19 \\
Kanto & 73 & 77 & 64 & 81 & 96 \\
Tokyo & 57 & 45 & 35 & 55 & 30 \\
Chubu & 53 & 48 & 31 & 46 & 49 \\
Kinki & 89 & 67 & 87 & 77 & 86 \\
Chugoku/Shikoku & 27 & 26 & 24 & 47 & 34 \\
Kyushu & 33 & 14 & 24 & 38 & 23 \\
Nation (total) & 386 & 341 & 320 & 390 & 351 \\
\hline
\end{tabular}

clinical effect of the standard multidrug regimen for the treatment of pulmonary tuberculosis [13]. The 2007 ATS/ IDSA statement recommended a multidisciplinary treatment approach to prevent disease progression: a combination of chemotherapy and adjuvant resectional surgery for patients with intractable NTM disease [3]. The JST guidelines also recommended this multidisciplinary treatment approach [4].

In this study, data from the JATS annual survey revealed a steady increase in the annual number of operations performed in Japan for pulmonary NTM disease. The overall number increased by $76 \%$ in 5 years. However, the annual number of operations for pulmonary tuberculosis was stable, as was the annual number of operations for tuberculoma in the country. The total number of general thoracic operations increased during the study period. However, the increase in the number of operations for pulmonary NTM disease cannot be explained simply by the increase in the number of total operations, because the ratio of the number of operations for pulmonary NTM disease to the total number of general thoracic operations also increased. The recent increase in the number of operations for pulmonary NTM disease may reflect the fact that the prevalence of pulmonary NTM disease has been increasing in Japan and that the 2007 ATS/IDSA statement and the 2008 JST guidelines have encouraged Japanese physicians to liberally apply adjuvant resectional surgery to patients with pulmonary NTM disease. This trend is expected to continue indefinitely. Japanese General Thoracic surgeons will continue to increasingly encounter NTM patients who are candidates for resectional surgery until a magic bullet against this intractable disease is available. A notable increase in the number of operations for pulmonary NTM disease was seen in six of the eight regions. Further investigation is needed to clarify a regional distribution of patients with pulmonary NTM disease in Japan.

Data from the JATS survey did not contain crucial information (e.g., as patient characteristics, including age, gender, NTM species, and disease type) that would have allowed a more precise analysis of the current status of NTM surgery. The data also lacked information on the duration of pre-operative chemotherapy, the type of pulmonary resection, and the duration of post-operative chemotherapy. Pulmonary MAC disease predominantly occurs in middle-aged women, and consists of two radiographically characteristic features: fibrocavitary disease and nodular/bronchiectatic disease [3]. Although MAC disease is the NTM disease most likely to require pulmonary resection, other less common NTM diseases, such as Mycobacterium abscessus disease, which is notoriously resistant to chemotherapeutic regimens, may also be 
subject to resectional surgery [14]. According to the JST guidelines, surgery should be performed after 3-6 months of chemotherapy in expectation of the limited but possible efficacy of chemotherapy and the reduction of bacterial load [4]. The procedure of choice is pulmonary resection; segmental or greater resection is warranted if either disseminated foci surround the affected lesions or if the lesions disseminate into airways because the lesions can extend through the airways. Chemotherapy should be continued for at least 1 year after surgery in accordance with the ATS/IDSA guidelines for medical therapy (i.e., for at least 1 year after sputum conversion) [3], and on the basis of previous experience. The National Clinical Database established in 2011 can provide information about a patient's age, gender, and type of pulmonary resection, but other relevant information remains missing. To perform a more in-depth analysis of the current status of NTM surgery, we need to conduct a national survey on this disease.

\section{Conclusions}

The JATS annual survey revealed a steady increase in the annual number of operations for pulmonary NTM disease in Japan. Japanese General Thoracic surgeons will continue to increasingly encounter NTM patients who are candidates for resectional surgery until a magic bullet against this intractable disease is available. A national survey focusing on this disease is needed for more in-depth analysis of the current status of NTM surgery.

Acknowledgments This work was supported in part by a grant from the Suzuken Memorial Foundation.

\section{Compliance with ethical standards}

Conflict of interest Yuji Shiraishi has no conflict of interest.

Open Access This article is distributed under the terms of the Creative Commons Attribution 4.0 International License (http://crea tivecommons.org/licenses/by/4.0/), which permits unrestricted use, distribution, and reproduction in any medium, provided you give appropriate credit to the original author(s) and the source, provide a link to the Creative Commons license, and indicate if changes were made.

\section{References}

1. Brode SK, Daley CL, Marras TK. The epidemiologic relationship between tuberculosis and non-tuberculous mycobacterial disease: a systematic review. Int J Tuberc Lung Dis. 2014;18:1370-7.

2. Morimoto K, Iwai K, Uchimura K, Okumura M, Yoshiyama T, Yoshimori $\mathrm{K}$, et al. A steady increase in nontuberculous mycobacteriosis mortality and estimated prevalence in Japan. Ann Am Thorac Soc. 2014;11:1-8.

3. Griffith DE, Aksamit T, Brown-Elliott BA, Catanzaro A, Daley C, Gordin F, et al. An official ATS/IDSA statement: diagnosis, treatment, and prevention of nontuberculous mycobacterial diseases. Am J Respir Crit Care Med. 2007;175:367-416.

4. Nontuberculous Mycobacteriosis Control Committee of the Japanese Society for Tuberculosis; International Exchanging Committee of the Japanese Society for Tuberculosis. Guidelines for surgical therapy for pulmonary nontuberculous mycobacterial diseases. Kekkaku. 2011;86:41-2.

5. Sakata R, Fujii Y, Kuwano H. Thoracic and cardiovascular surgery in Japan during 2008: annual report by The Japanese Association for Thoracic Surgery. Gen Thorac Cardiovasc Surg. 2010;58:356-83.

6. Sakata R, Fujii Y, Kuwano H. Thoracic and cardiovascular surgery in Japan during 2009: annual report by The Japanese Association for Thoracic Surgery. Gen Thorac Cardiovasc Surg. 2011;59:636-67.

7. Kuwano H, Amano J, Yokomise H. Thoracic and cardiovascular surgery in Japan during 2010: annual report by The Japanese Association for Thoracic Surgery. Gen Thorac Cardiovasc Surg. 2012;60:680-708.

8. Amano J, Kuwano H, Yokomise H. Thoracic and cardiovascular surgery in Japan during 2011: annual report by The Japanese Association for Thoracic Surgery. Gen Thorac Cardiovasc Surg. 2013;61:578-607.

9. Masuda M, Kuwano H, Okumura M, Amano J, Arai H, Endo S, et al. Thoracic and cardiovascular surgery in Japan during 2012: annual report by The Japanese Association for Thoracic Surgery. Gen Thorac Cardiovasc Surg. 2014;62:734-64.

10. Sakatani M. The non-tuberculous mycobacteriosis [in Japanese]. Kekkaku. 2005;80:25-30.

11. American Thoracic Society. Diagnosis and treatment of disease caused by non-tuberculous mycobacteria. Am J Respir Crit Care Med. 1997;156:S1-25.

12. Atypical Mycobacteriosis Control Committee of the Japanese Society for Tuberculosis. Opinions of Japanese Society for Tuberculosis committee on therapy of atypical mycobacterium infections [in Japanese]. Kekkaku. 1998;73:599-605.

13. Kobashi Y, Matsushima T. The effect of combined therapy according to the guidelines for the treatment of Mycobacterium avium complex pulmonary disease. Intern Med. 2003;42:670-5.

14. Griffith DE. The talking Mycobacterium abscessus blues. Clin Infect Dis. 2011;52:572-4. 